\title{
Irreversible aggregation of flexible chainlike walkers without adherence
}

\author{
Takashi Mashiko* \\ Department of Mechanical Engineering, Shizuoka University, Hamamatsu 432-8561, Japan \\ (Received 16 March 2008; revised manuscript received 16 May 2008; published 3 July 2008)
}

\begin{abstract}
The flexible chainlike walker (FCW) model is proposed as a minimal model of a deformable moving object and as an extension of the regular random-walk model. The many-body system of FCWs is studied by numerical simulations on a square lattice. It is shown that FCWs aggregate spontaneously and irreversibly where no adherence is assumed, in contrast to the established aggregation models, where adherence is indispensable for their occurrence and irreversibility. This type of aggregation is enabled by and demonstrates the significance of the deformability of moving objects.
\end{abstract}

DOI: 10.1103/PhysRevE.78.011106

PACS number(s): 05.40.Fb, 45.50.Jf, 61.43.Hv

The concept of random walk has played important roles in a wide variety of disciplines, such as economics, population genetics, polymer science, and basic physics. Much is known about the behaviors of a single random walker [1,2]. Also, systems in which multiple walkers are involved have attracted considerable attention. For instance, the territory visited by many random walkers $[3,4]$ and aggregation processes of numerous random walkers [5-11] have been studied. In the studies of many-body systems of random walkers, however, the "walker" has been treated just as a point (occupying a single site in an on-lattice case), or as a rigid body (occupying multiple sites), if not pointlike. For example, in modeling aggregation processes of randomly moving objects, each object has been treated as a pointlike particle [5-9] or an undeformable aggregate of particles $[10,11]$. Likewise, in studying pedestrian or vehicular traffic flows using a "biased" random-walk model, each locomotor has been mimicked by a point $[12,13]$ or by a rod [14]. It may be said that such a pointlike-body or rigid-body approximation has been tacitly assumed to be of no importance or simply accepted without much attention in studying many-body systems of moving objects, whereas various models have been proposed for a single moving object with deformability [15-21].

From the point of view of statistical physics, it is important and intriguing to examine how the microscopic, individual properties of the moving object affect the macroscopic, collective behaviors. Also, from the standpoint of application potentiality, it is interesting to investigate the properties of deformable moving objects, since there are actually various moving objects that are deformable around us. Even for one-dimensional objects alone, examples can be given from those living, such as some kinds of bacteria and worms, to those nonliving, such as container dollies towed by a tractor and snakelike robots. In particular, studying the behaviors of deformable machines is of increasing importance, in view of the rapid factory automation and the recent development of snakelike robots [22]. Therefore, it is a worthwhile issue to study the effect of the deformability of moving objects.

\footnotetext{
*ttmasik@ipc.shizuoka.ac.jp
}

In this paper, a flexible chainlike walker (FCW) model is proposed for randomly moving objects possessing deformability, and the collective behavior of the many-body system of FCWs is examined through numerical simulations on a square lattice. It is shown that FCWs exhibit a novel type of irreversible aggregation, despite the lack of adherence, which has always been assumed in previous studies of aggregation processes. The irreversibility proves to be an outcome of the deformability of FCWs.

The following is a description of the FCW model: An FCW of length $l$ is represented by $l$ serially concatenated particles, which, on a square lattice, occupy $l$ horizontally or vertically adjacent sites. One of the edge particles (the first particle) represents the head of the FCW and the other (the $l$ th particle) represents the tail. Figure 1 schematically exemplifies the movement of an $l=5 \mathrm{FCW}$. At each time step, the head particle (represented by the double circle) chooses one of its four nearest-neighbor sites at random and moves to that site if it is not occupied by another particle (either of the same FCW or of another). Then the subsequent particles follow the head particle. That is, the second particle moves to the site that the head particle has just left, the third particle moves to the site that the second particle has just left, and so forth. If the site chosen by the head particle is occupied, the FCW does not move at that time step.

As shown before, the FCW model has a single free parameter, namely the length $l$. Also, the regular random walk, in which the pointlike walker moves to one of the nearest-

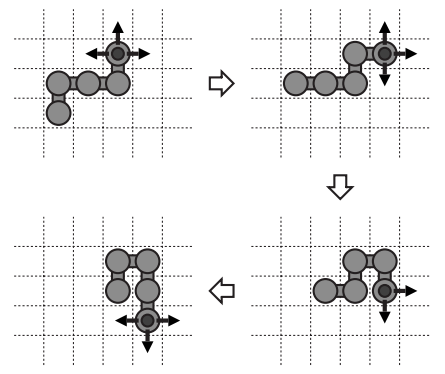

FIG. 1. Flexible chainlike walker model on a square lattice. A typical series of movements of an $l=5 \mathrm{FCW}$ is illustrated. At each time step, the head particle (represented by the double circle) chooses one of its four nearest-neighbor sites at random and moves to that site if it is vacant, followed by the subsequent particles. Possible moving directions are indicated by the arrows. 


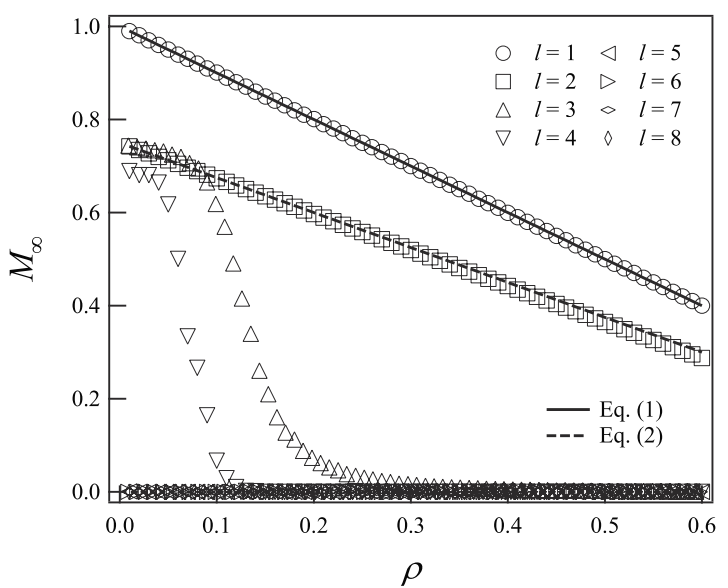

FIG. 2. Asymptotic mobility $M_{\infty}$ as a function of particle density $\rho$. Numerical results are shown for $1 \leqslant l \leqslant 8$, together with the theoretical predictions for $l=1$ and 2 [Eqs. (1) and (2), respectively]. Each symbol represents the averaged value taken over 50000 time steps in the asymptotic state and over at least 10 runs.

neighbor sites at every time step, irrespective of its track, is recovered from the FCW model in the limit of $l=1$. In addition, it is apparent that an FCW of $l=2$ does not change its shape (but just changes direction), whereas an FCW of $l \geqslant 3$ can deform.

In the present study, $N$ FCWs are placed on a square area of $W \times W$ sites. To describe the behaviors of FCWs, the following quantities are defined: The density of particles is the ratio of the total number of particles to the number of sites on the square area; $\rho=l N / W^{2}$. The mobility of FCWs at time $t$ is defined as $M(t)=N_{\text {mov }}(t) / N$, where $N_{\text {mov }}(t)$ is the number of FCWs that have succeeded in moving at that time step. At $t=0$, FCWs are placed at random positions as initial distribution. Each FCW is put straight horizontally. This is partly for simplicity and partly for avoiding inborn "locking" (explained below). Then the FCWs are updated following the above-described rule in random order at every time step. In the present work, simulations are conducted with $W=100$ under periodic boundary condition.
It was found that the mobility $M$ decreases with time, to an asymptotic value $M_{\infty}=M(t \rightarrow \infty)$. Typically, $t \sim 50000$ was enough for $M$ to reach $M_{\infty}$, except for $l=3$, in which case slower convergence was observed. In Fig. 2, the values of $M_{\infty}$, each of which is averaged over 50000 time steps in the asymptotic state (e.g., $t=50001-100000$ ) and over at least 10 runs, are plotted as functions of $\rho$ for $1 \leqslant l \leqslant 8$, together with two theoretical lines,

$$
\begin{gathered}
M_{\infty}=1-\rho, \\
M_{\infty}=\frac{3}{4}(1-\rho) .
\end{gathered}
$$

These formulas are derived from the following straightforward discussion: For $l=1$, an FCW, or the regular random walker, is a single particle. The probability of occupation of a site does not depend on whether the site is a nearest neighbor of an FCW, provided $N$ is sufficiently large. Thus the mobility (i.e., the probability that the nearest-neighbor site chosen by an FCW is empty) is equal to $1-\rho$, and Eq. (1) is derived for $l=1$. In the case of $l=2$, an FCW consists of a head particle and a tail particle. Hence, one of the four nearest-neighbor sites of the head particle of an FCW is always occupied by its own tail particle, and each of the other three nearest-neighbor sites is occupied with the probability of $1-\rho$, as in the case of $l=1$. Therefore, we obtain Eq. (2) for $l=2$. Figure 2 shows that the numerical results for $l=1$ and 2 agree with the theoretical predictions. On the other hand, the results for $l=3$ and 4 show sharp drops from the higher- $M_{\infty}$ state to the $M_{\infty} \sim 0$ state. In addition, $l=5$ or longer FCWs settle down in the $M_{\infty} \sim 0$ state even when the density $\rho$ is low.

To clearly demonstrate the difference between the rigid, shorter FCWs $(l \leqslant 2)$ and the deformable, longer FCWs $(l \geqslant 3)$, typical pattern variations with time of $l=2$ and 3 FCWs are shown in Figs. 3(a) and 3(b), respectively. The density is the same for both cases; $\rho=0.5$ (the number of FCWs is $N=2500$ for $l=2$ and $N=1667$ for $l=3$ ). The snapshots are of $t=0$ (initial distribution), 1000, 2000, 3000, 10000 , and 100000 (from left to right). The difference is

(a)
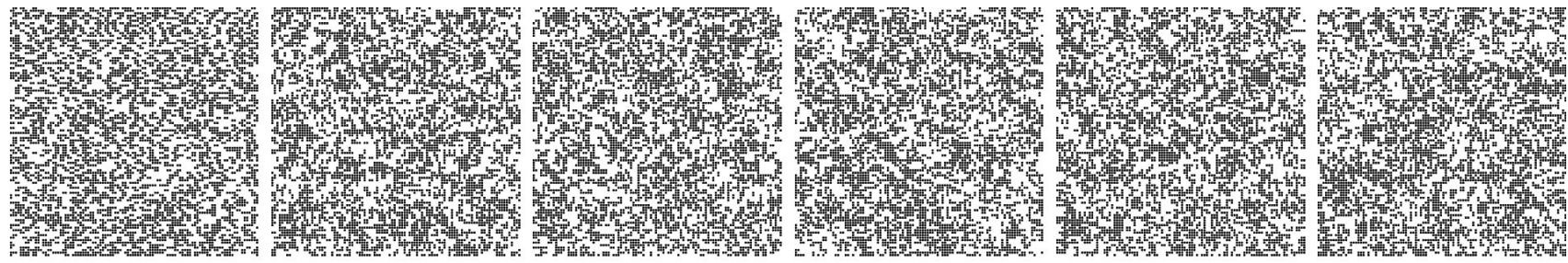

(b)
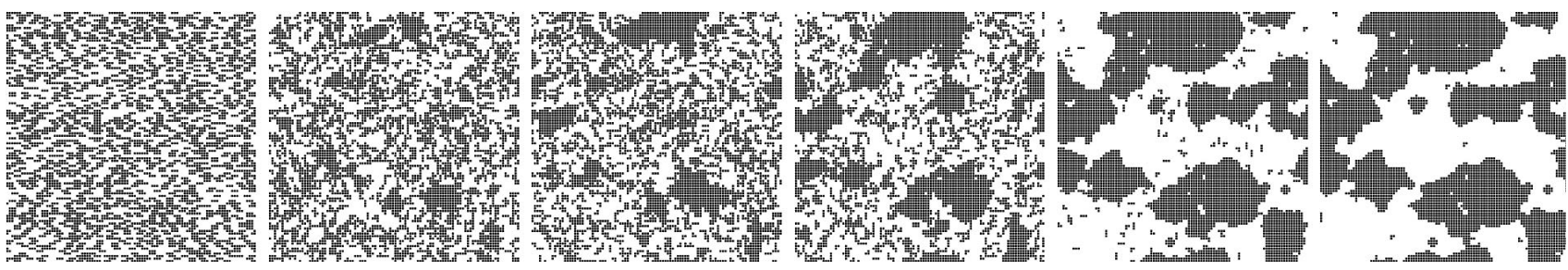

FIG. 3. Typical time variations of the FCW distributions for $\rho=0.5$ on a square area of $W=100$ on a side. (a) $l=2$ FCWs $(N=2500)$. (b) $l=3$ FCWs $(N=1667)$. Snapshots at $t=0,1000,2000,3000,10000$, and 100000 (from left to right) are shown. 
(a)
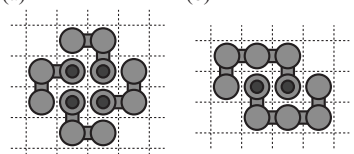

(c)

(d)

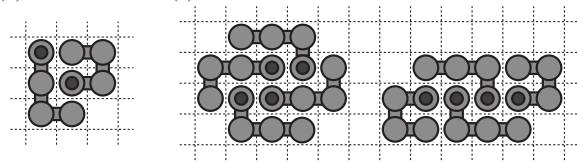

FIG. 4. Examples of mutual locking of FCWs. (a) Four-body mutual locking of $l=3$ FCWs. (b) Two-body mutual locking of $l=5$ FCWs. (c) Self-locking of an $l=8$ FCW. (d) Quasi-mutuallocking of $l=4 \mathrm{FCWs}$, where the right one is locked by the left one, while the left one is not completely locked by the right one (but by other FCWs, which are not shown here). (e) Other types of fourbody mutual locking than type (a), possible for $l=4$ FCWs.

apparent: $l=3$ FCWs aggregate irreversibly, whereas $l=2$ FCWs show no irreversible aggregation and no apparent changes with time.

What causes this crucial difference? Observing the time variations in detail, we notice that $l=3$ or longer FCWs sometimes form a scrum when they happen to encounter each other, and get locked by themselves. That is, all four nearest-neighbor sites of the FCWs' head particles are occupied by their own particles. Let us refer to such a situation as "mutual locking." Some examples of mutual-locking patterns are shown in Fig. 4: Four $l=3$ or longer FCWs can form a fylfot scrum (a). FCWs of $l=5$ or longer can form couplershaped gridlock when two of them happen to meet (b). Furthermore, if an FCW is long enough $(l \geqslant 8)$, it can lock itself by coiling (c), which is called here "self-locking." In some cases, the locking is not completely mutual, as exemplified in (d), which is, so to speak, "quasi-mutual-locking." Selflocking and quasi-mutual-locking, however, are regarded here as special cases of mutual locking. Once mutual locking takes place, the FCWs concerned cannot move anymore. This causes the irreversibility of the aggregation process. On the other hand, shorter FCWs of $l=1$ or 2 do not go into mutual locking, no matter how many FCWs happen to gather at once.

The above discussion qualitatively explains the basic features observed in Fig. 2: FCWs of $l=1$ and 2, which do not undergo mutual locking, never aggregate irreversibly, and hence the simple theoretical predictions of Eqs. (1) and (2) stand, respectively. In the case of $l=3$, the same theory as $l$ $=2$ [i.e., Eq. (2)] holds when the density $\rho$ is low, since four-body collisions seldom take place. For high $\rho$ values, on the other hand, they come to aggregate irreversibly, nucleated by mutual locking, and accordingly the mobility decreases with time, until it finally approaches $M_{\infty} \sim 0$. Therefore, there is a transition from a moving (higher- $M_{\infty}$ ) state to an aggregating $\left(M_{\infty} \sim 0\right)$ state. Similar discussion holds for $l=4$, with small differences in the values of $M_{\infty}$ in the moving state and in the density at which the transition occurs. The former difference is due to the fact that one of the nearest-neighbor sites of the head particle of an FCW can be occupied by the fourth (tail) particle of the same FCW, in addition to the second particle which always occupies one nearest-neighbor site. The latter difference is brought about by the fact that some types of four-body mutual locking, such as those illustrated in Fig. 4(e), are possible for $l=4$ FCWs, in addition to the type of Fig. 4(a), thus they are more likely to lock themselves than $l=3$ FCWs. It is easy for much longer FCWs $(l \geqslant 5)$ to aggregate irreversibly even for low $\rho$, since mutual locking by only two FCWs or the self-locking can take place. Hence, they settle down in the $M_{\infty} \sim 0$ state even for low $\rho$.

The FCW model itself is not necessarily unique, since there have been several similar models of polymer chain or generalizations of the random walk. For example, the reptation model [15], self-avoiding walk (SAW), true SAW (TSAW) [16], indefinitely growing SAW (IGSAW) [17], smart kinetic walk (SKW) [18], Laplacian random-walk (LRW) [19,20], and the kinetic growth walk (KGW) [21] have been proposed, and their behaviors have been investigated and contrasted. In addition, in some of the models such as SAW or KGW, self-trapping behaviors are pointed out, which are inherently the same as the above-mentioned "selflocking." However, the point of interest in the present work is the collective behavior of the many-body system of walkers, whereas the main target in the above-mentioned works is the kinetics of a growing single walker. It is obvious that the (narrowly defined) mutual locking (excluding self-locking) and the consequent irreversible aggregation without adherence are outcomes that can be obtained only when multiple walkers are concerned.

The novelty of the aggregation mechanism presented in this paper should be emphasized here. There have been several models of aggregation processes, from those of pointlike particles $[5-9,23-25]$ to those of particles aggregates $[10,11,26]$. In all these established models, some adherence has been assumed. That is, in terms of a particle-aggregation process such as the well-known diffusion-limited aggregation (DLA) [6] or ballistic deposition [23,25], a particle adheres to and becomes part of an existing aggregate when it comes in contact with the aggregate, even if stochastically (i.e., with a sticking probability $p>0$ ). Also, it is easy to see that adherence is virtually assumed in somewhat different types of aggregation models such as the Eden model [27], which was originally proposed for the growth process of a cell colony, where one of the adjoining sites of the colony (aggregate) is chosen and occupied by a new cell (particle). Obviously, in all these models, adherence is essential for the process of aggregation and the irreversibility is due to this adherence. In the present model, on the other hand, FCWs aggregate spontaneously and irreversibly, though no such adherence is assumed (i.e., $p=0$ ). Adherence is not required for the occurrence of aggregation and the irreversibility results from the mutual locking of FCWs. Therefore, the aggregation mechanism of FCWs is fundamentally different from that of the conventional models. The fact that this novel type of aggregation is possible only for the many-body system of deformable objects strongly suggests the significance of studying such a system as in the present work.

To conclude, in the present paper, a flexible chainlike walker (FCW) model has been proposed, and an irreversible aggregation process of FCWs without adherence has been 
demonstrated by numerical simulations. This novel type of aggregation occurred only for $l \geqslant 3$, which clearly indicates that it is enabled by the deformability of FCWs. Further studies of many-body systems of FCWs, motivated by the present work, would be challenging. For example, extending this model for off-lattice or higher-dimensional space may reveal some other properties peculiar to deformable objects. Also, it is intriguing to apply the idea of this model to other models. To give an example, the patterns and statistical prop- erties of DLA could be changed when the diffusing pointlike particles are replaced by FCWs. Likewise, application of the FCW model to collective motions of self-driven objects $[28,29]$ and pedestrian or vehicular traffic flows [12-14,30] would be interesting. Some studies of traffic flows using a "biased" FCW model are actually in progress and will be reported elsewhere [31,32].

The author thanks T. Nagatani for useful discussions.
[1] J. W. Cohen, Analysis of Random Walks (IOS, Tokyo, 1992).

[2] B. D. Hughes, Random Walks (Clarendon, Oxford, 1995).

[3] H. Larralde, P. Trunfio, S. Havlin, H. E. Stanley, and G. H. Weiss, Nature 355, 423 (1992).

[4] E. Arapaki, P. Argyrakis, and A. Bunde, Phys. Rev. E 69, 031101 (2004).

[5] H. B. Rosenstock and C. L. Marquardt, Phys. Rev. B 22, 5797 (1980).

[6] T. A. Witten, Jr. and L. M. Sander, Phys. Rev. Lett. 47, 1400 (1981).

[7] P. Meakin, Phys. Rev. A 27, 604 (1983).

[8] M. Matsushita and H. Kondo, J. Phys. Soc. Jpn. 55, 2483 (1986).

[9] M. Matsushita, K. Honda, H. Toyoki, Y. Hayakawa, and H. Kondo, J. Phys. Soc. Jpn. 55, 2618 (1986).

[10] P. Meakin, Phys. Rev. Lett. 51, 1119 (1983).

[11] M. Kolb, R. Botet, and R. Jullien, Phys. Rev. Lett. 51, 1123 (1983).

[12] M. Muramatsu, T. Irie, and T. Nagatani, Physica A 267, 487 (1999).

[13] M. Isobe, D. Helbing, and T. Nagatani, Phys. Rev. E 69, 066132 (2004).

[14] R. Nagai and T. Nagatani, Physica A 366, 503 (2006).

[15] P. G. de Gennes, J. Chem. Phys. 55, 572 (1971).

[16] D. J. Amit, G. Parisi, and L. Peliti, Phys. Rev. B 27, 1635 (1983).

[17] K. Kremer and J. W. Lyklema, Phys. Rev. Lett. 54, 267
(1985).

[18] A. Weinrib and S. A. Trugman, Phys. Rev. B 31, 2993 (1985).

[19] J. W. Lyklema, C. Evertsz, and L. Pietronero, Europhys. Lett. 2, 77 (1986).

[20] J. W. Lyklema and C. Evertsz, J. Phys. A 19, L895 (1986).

[21] I. Majid, N. Jan, A. Coniglio, and H. E. Stanley, Phys. Rev. Lett. 52, 1257 (1984)

[22] A. A. Transeth and K. Y. Pettersen, Proceedings of the IEEE International Conference on Control, Automation, Robotics, and Vision (IEEE, Piscataway, NJ, 2006), p. 1393.

[23] M. J. Vold, J. Colloid Sci. 18, 684 (1963).

[24] H. P. Hutchinson and D. N. Sutherland, Nature 206, 1036 (1965).

[25] P. Meakin, P. Ramanlal, L. M. Sander, and R. C. Ball, Phys. Rev. A 34, 5091 (1986).

[26] D. N. Sutherland, Nature 226, 1241 (1970).

[27] M. Eden, Proceedings of the Fourth Berkeley Symposium on Mathematical Statistics and Probability (University of California Press, Berkeley, 1961), Vol. 4, p. 223.

[28] T. Vicsek, A. Czirók, E. Ben-Jacob, I. Cohen, and O. Shochet, Phys. Rev. Lett. 75, 1226 (1995).

[29] N. Shimoyama, K. Sugawara, T. Mizuguchi, Y. Hayakawa, and M. Sano, Phys. Rev. Lett. 76, 3870 (1996).

[30] D. Helbing, I. Farkas, and T. Vicsek, Nature 407, 487 (2000).

[31] T. Mashiko, Y. Imanishi, R. Kuwajima, and T. Nagatani (unpublished).

[32] A. Matsui, T. Mashiko, and T. Nagatani (unpublished). 